\title{
Simalikalactone E (SkE), a new weapon in the armamentarium of drugs targeting cancers that exhibit constitutive activation of the ERK pathway
}

\author{
Guillaume Robert',2,3,4, Valérie Jullian ${ }^{6,7}$, Arnaud Jacquel 1,2,3,4, Clémence Ginet ${ }^{1,2,3,4}$, \\ Maeva Dufies ${ }^{1,2,3,4}$, Stephanie Torino ${ }^{1,4,5}$, Anaïs Pottier ${ }^{1,4,8}$, Frederic Peyrade ${ }^{9}$, Sophie \\ Tartare-Deckert ${ }^{1,4,8}$, Geneviève Bourdy ${ }^{6,7}$, Eric Deharo ${ }^{6,7}$ and Patrick Auberger ${ }^{1,2,3,4}$

Copyright: () Robert et al. This is an open-access article distributed under the terms of the Creative Commons Attribution License, which permits unrestricted use, distribution, and reproduction in any medium, provided the original author and source are credited.

\section{ABSTRACT:}

Simalikalactone E (SkE) is a quassinoid extracted from a widely used Amazonian antimalarial remedy. Although SkE has previously been shown to have cytostatic and/or cytotoxic activities in some tumor cell lines, its mechanism of action has not yet been characterized. We show here that SkE in the high nanomolar range inhibited the growth of various leukemic and solid tumor cell lines. Importantly, SkE was highly efficient at inhibiting chronic myelogenous leukemia (CML) cells that exhibit constitutive activation of the MAPK pathway and, accordingly, it impaired the phosphorylation of ERK1/2. SkE also abrogated MEK1/2 and B-Raf phosphorylation but had no effect on Ras activity. Moreover, SkE was particularly effective against melanoma cell lines carrying the B-Raf-V600E mutation. Importantly, SkE resensitized the PLX-4032-resistant 451Lu melanoma cell line (451Lu-R) and was more efficient than U0126, a MEK inhibitor, and PLX-4032 (PLX) at inducing the apoptosis of two Hairy Cell Leukemia (HCL) patient samples carrying the B-Raf-V600E mutation. Finally, SkE was as efficient as imatinib at inhibiting tumor formation in a xenograft model of CML cells in athymic mice. In conclusion, we show that SkE, a very potent inhibitor of B-Raf-V600E, is highly effective against cancer cell lines that exhibit constitutive activation of the ERK1/ 2 pathway.

\section{INTRODUCTION}

Simalikalactone E (SkE) is a new quassinoid extracted from a widely used Amazonian antimalarial remedy derived from Quassia amara L. (Simaroubaceae) leaves. In the mid-nanomolar concentration range, this new molecule inhibits the growth of Plasmodium falciparum cultured in vitro by $50 \%$, independent of the strain sensitivity to chloroquine. SkE can also decrease gametocytemia when present at a 50\% inhibitory concentration seven fold lower than that of primaquine, a 
leading compound for treating malaria. SkE is less toxic than simalikalactone $\mathrm{D}(\mathrm{SkD})$, another antimalarial related quassinoid from Quassia amara, and its cytotoxicity towards mammalian cells is dependent on the cell line; it displays a good selectivity index when tested on nontumorigenic cells. In vivo, SkE inhibits murine malarial growth of Plasmodium vinckei petteri by $50 \%$ at doses of 1 and $0.5 \mathrm{mg} / \mathrm{kg}$ body weight/day when administered by the oral and intraperitoneal route, respectively [1]. Furthermore, unpublished data from our laboratories have established that $\mathrm{SkE}$ may have potent antileukemic activity on several hematological malignancies.

The Ras/Raf/MEK/ERK pathway is frequently altered in cancer cells, and mutations in this pathway are recurrent in several hematopoietic and non-hematopoietic malignancies [2, 3]. It is also worth mentioning that mutation of an upstream protein in the MAP kinase pathway excludes the possibility of mutation of another protein in the pathway $[4,5]$. For instance, N-Ras, one of the upstream regulators of the pathway, is mutated in $20 \%$ of melanoma, whereas K-Ras is mutated in $80 \%$ of pancreatic carcinoma. B-Raf, an effector of Ras and the upstream kinase in the ERK cascade, is frequently mutated in melanoma (50-70\%) [6], Langerhans cell histiocytosis (57\%) [7], thyroid carcinoma (40\%) [8] and colorectal cancer (8\%) [9]. The frequency of B-Raf mutation is generally very low in leukemia; however, it was recently reported that B-Raf is mutated in most cases of HCL [10-12]. Finally, mutations in MEK1 are also detected at a low frequency in melanoma [13]. In all cases, the mutated protein seems to be endowed with constitutive activity. Inhibitors of B-Raf such as PLX have been introduced recently with success as new anti-melanoma agents that can induce complete remission in patients [14]. Unfortunately, resistance to PLX has been found to occur rapidly after the onset of treatment, mainly through reactivation of the MAP kinase pathway [15]. Therefore, it is essential to develop new therapeutic strategies aimed at inhibiting the MAPK pathway in these resistant patients.

Importantly, HCL is another disease characterized by the B-Raf mutation [10]. HCL is a rare leukemia affecting B cells. This hematopoietic malignancy is associated with the B-Raf V600E mutation in most of patients. This hallmark of the disease has provided the rationale for the use of vemurafenib (PLX-4032) in two patients suffering from HCL who had no other therapeutic options [16]; Peyrade et al. 2012 (in press). In both cases, a two-month treatment with the drug led to elimination of the leukemic clone as well as restoration of normal erythrocyte, platelet and leukocyte counts, which were accompanied by a considerable improvement in the patient status.

In the present study, we describe the activity and mechanism of action of SkE, a new natural compound extracted from Quassia Amara that exhibits both potent anti-leukemic and anti-melanoma effects in vitro and in vivo because of its ability to interfere with the ERK cascade. Therefore, SkE should be tested as a new therapeutic option in cancers that exhibit constitutive activation of the ERK pathway.

\section{RESULTS}

\section{SkE exerts potent antileukemic activity in vitro}

We have reported previously that $\mathrm{SkE}$ is both cytostatic and cytotoxic for some tumor cell lines [1]. The present study was conducted to address the mechanism of action of SkE in different cancer cell lines. We first used the well-characterized human K562 cell line to determine whether SkE affects the proliferation of leukemic cells. To this end, we performed colony formation assays in soft-agar using increasing doses of $\mathrm{SkE}$ or a maximal dose of imatinib, a tyrosine kinase inhibitor that targets $\mathrm{BCR}-\mathrm{ABL}$, the fusion oncoprotein responsible for this disease. As expected, imatinib $(1 \mu \mathrm{M})$ inhibited the clonogenic potential of K562 cells in soft-agar by more than $90 \%$ (Figure 1A). Importantly, SkE was a highly potent inhibitor of K562 cell colony formation in identical conditions, with a maximal effect at $500 \mathrm{nM}$ (Figure 1A). At this dose, SkE was even more potent than imatinib, the leading therapy for CML. The IC50 value for the SkE effect was found to be $250 \mathrm{nM}$. SkE was also a very potent inhibitor of CD34+ cell growth for cells isolated from two CML patients at diagnosis (Figure 1B). Finally, SkE also exerted potent antileukemic effects on several imatinibresistant CML cell lines (not shown).

\section{SkE inhibits the MAP kinase pathway}

In an attempt to identify the potential targets of SkE, we used the PathScan ${ }^{\circledR}$ RTK signaling antibody array kit from Cell Signaling, which allows the simultaneous quantification of the activity of approximately 50 kinases. Among these kinases, two were significantly affected by SkE. Indeed, SkE inhibited the activity of ERK by $70 \%$ and $\mathrm{c}-\mathrm{Abl}$ by $15 \%$ (Figure $2 \mathrm{~A}$ and $\mathrm{B}$ ). To confirm the effect of SkE on BCR-ABL activity, we next incubated K562 cells for $2 \mathrm{~h}$ with $250 \mathrm{nM}$ of SkE and analyzed the phosphorylation status of both BCR-ABL and known BCR-ABL substrates. In accordance with the results obtained with the RTK signaling array kit, we confirmed the inhibition of c-Abl by $\mathrm{SkE}$ as judged by the decreased phosphorylation of c-Abl as soon as $3 \mathrm{hrs}$ after the addition of SkE to the culture medium. We also noted a decrease in the phosphorylation status of STAT5 (Figure 2C). Moreover, dephosphorylation of ERK1/2 was clearly detected as soon as $30 \mathrm{~min}$ after the addition of $\mathrm{SkE}$ and was maximal at $15 \mathrm{~h}$. Collectively, our results confirm that $\mathrm{SkE}$ is a very potent inhibitor of the ERK pathway in K562 
cells. Furthermore, it appears that c-Abl dephosphorylation did not precede ERK dephosphorylation but rather followed ERK inhibition. Figure $2 \mathrm{C}$ also shows that $\mathrm{SkE}$ failed to affect autophagy in K562 CML cells, as assessed by the absence of delipidation of LC3-b in cells treated with this drug.

\section{SkE is a B-Raf inhibitor}

We next used the $\Delta$ Raf-1:ER (HEK-ER) cells, which express an inducible form of the kinase Raf-1, to assess the effects of SkE in comparison with U0126, a well-known inhibitor of MEK1, in the Ras/Raf/MEK/ERK pathway. Tamoxifen induced the activation of the ERK pathway, as assessed by the increased phosphorylation of ERK1/2 (Figure 3A). Importantly, SkE (500 nM) was as efficient as U0126 $(2.5 \mu \mathrm{M})$ at abolishing tamoxifen-induced ERK1/2 activation (Figure $3 \mathrm{~B}$ ). To precisely identify the target of SkE, we analyzed the entire ERK pathway. SkE efficiently inhibited the phosphorylation status of both MEK1/2 and B-Raf (Figure 3C). However, SkE failed to affect the activity of Ras in a GST-RAS pull-down assay (Figure 3D). Collectively, our data clearly demonstrate that SkE

A

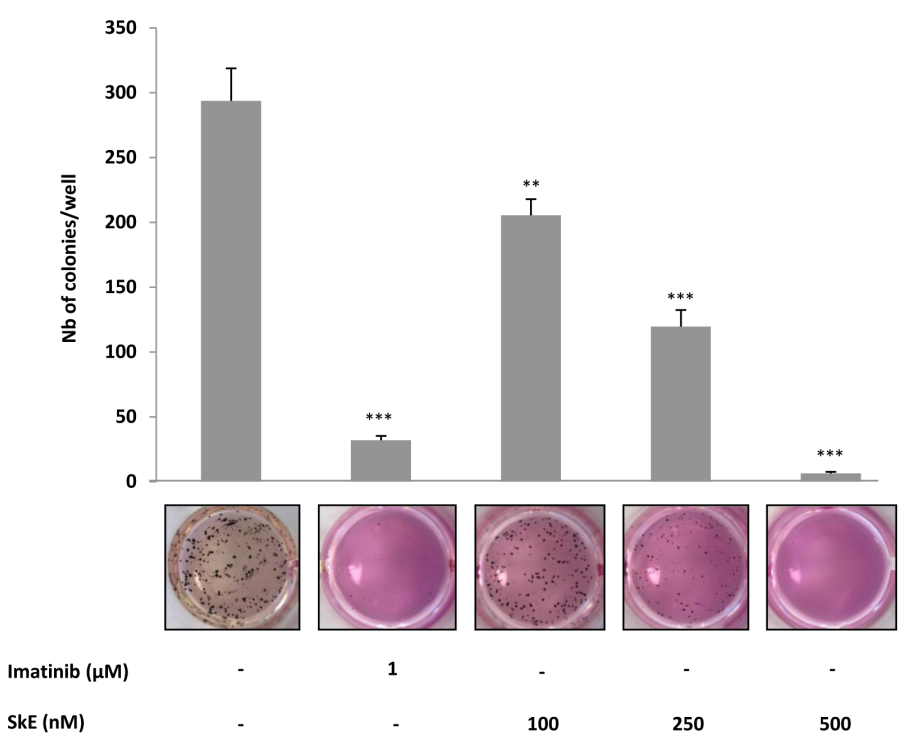

B

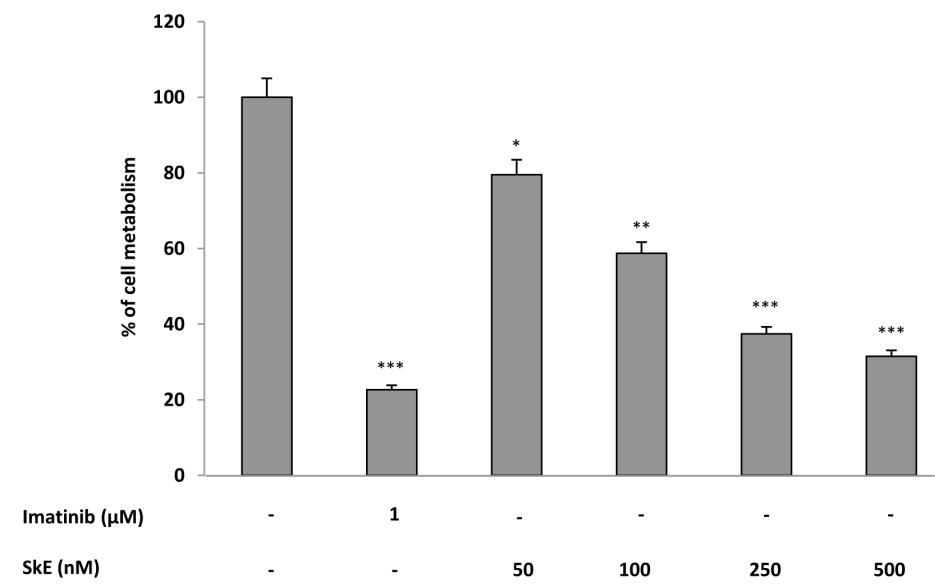

Figure 1: SkE treatment induces cell death of CML cell lines and primary CML CD34+ cells. (A) SkE in the 100-500 nM range was added to K562 CML cell lines growing in semi-solid methyl cellulose medium $\left(0.5 \times 10^{3}\right.$ cells $\left./ \mathrm{ml}\right)$. Imatinib $(1 \mu \mathrm{M}) \mathrm{was}$ used as an internal control. Colonies were detected after 10 days of culture by adding $1 \mathrm{mg} / \mathrm{ml}$ of MTT reagent and were scored by Image J quantification software. Results are expressed as the number of colony forming cells per well after drug treatment. Results are given as the mean \pm SD of 3 different determinations made in triplicate. Error bars $=95 \%$ confidence intervals. (B) Primary CML CD34+ cells were incubated for $48 \mathrm{~h}$ at $37^{\circ} \mathrm{C}$ with increasing concentrations of SkE. The cell metabolism was measured by the XTT assay, as described in the Materials and Methods section. Results are given as the mean \pm SD of 3 different determinations made in triplicate. Error bars $=95 \%$ confidence intervals. 
acts as an inhibitor of B-Raf. Finally, the effect of SkE on the ERK cascade was rapidly reversible upon withdrawal of the drug (Figure 3E).

\section{SkE inhibits the growth of PLX resistant-cell lines in vitro}

PLX, also known as vemurafenib, has been shown to be highly effective in both B-Raf V600E melanoma cell

A

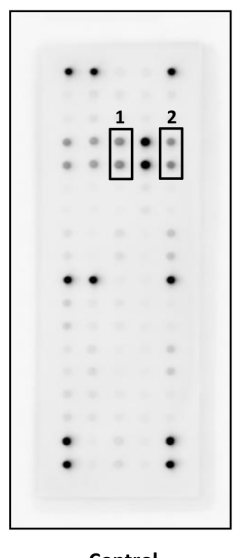

Control

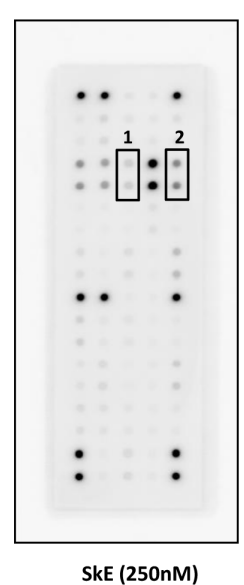

SkE (250nM) lines and in patients with metastatic melanoma. However, in patients, the rapid reactivation of the ERK cascade is responsible for relapses. We investigated whether $\mathrm{SkE}$ was capable of resensitizing PLX-resistant cell lines. To this end, we used dabrafenib (GSK2118436) sensitive and resistant melanoma cell lines which also exhibits cross resistance to vemurafenib (PLX-4720). This PLXsensitive 451 melanoma cell line and its PLX-resistant counterpart were incubated for $24 \mathrm{~h}$ with PLX $(1 \mu \mathrm{M})$

B

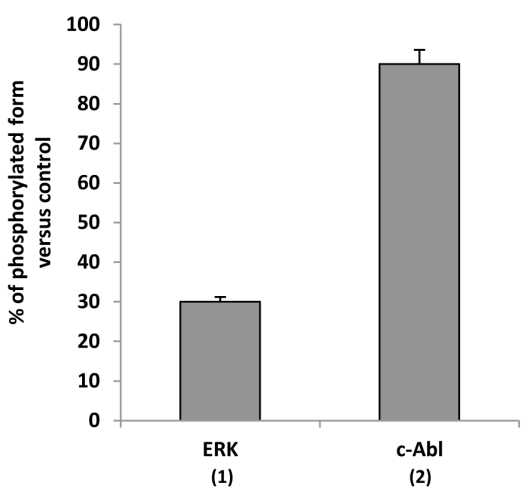

C

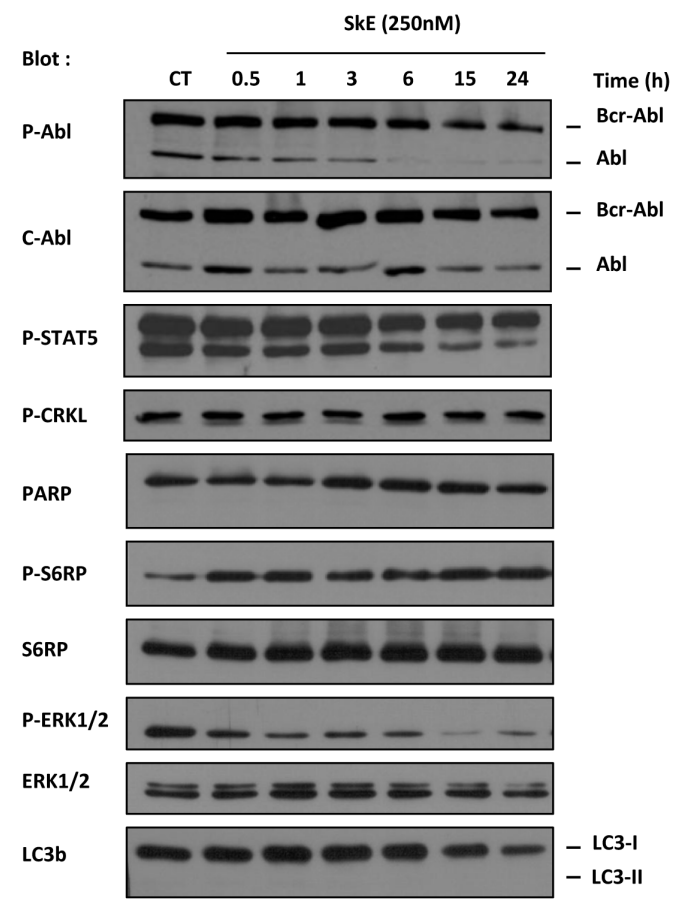

Figure 2: SkE treatment impairs ERK1/2 phosphorylation. (A) K562 cells were treated with $250 \mathrm{nM}$ of SkE for $2 \mathrm{~h}$; then, cells were lysed, and cell lysates were loaded on a Pathscan multikinase ${ }^{\circledR}$ membrane. (B) Histograms represent the relative intensity quantification of the most regulated dot with Image J software. Results are expressed as the percentage of kinase phosphorylation in SkEtreated cells versus control cells. (C) K562 cells were incubated at $37^{\circ} \mathrm{C}$ with $250 \mathrm{nM} \mathrm{SkE}$ for the indicated times. Whole-cell lysates were prepared, and the expression of Phospho-C-Abl, C-Abl, Phospho-STAT5, Phospho-CRKL, PARP, Phospho-S6RP, S6RP, PhosphoERK1/2, ERK1/2 and LC3b was visualized on a Western blot. 
or two concentrations of $\mathrm{SkE}$ and the cell viability was assessed using the XTT assay. As expected, the 451Lu$\mathrm{R}$ melanoma cell lines [22] were fully resistant to PLX, whereas both the 451Lu-R cell lines were highly sensitive to the effect of $\mathrm{SkE}$ (Figure 4A). Importantly, PLXresistant cells appeared to be even more sensitive to SkE.

We next analyzed the efficiency of U0126, PLX and SkE on blood cells from two HCL patients carrying the B-Raf V600E mutation. SkE, at a concentration of $500 \mathrm{nM}$, induced cell death in more than $70 \%$ of the blood cells, as assessed by propidium iodide staining (Figure 4B), whereas PLX and U0126 were less efficient, triggering $55 \%$ and $44 \%$ cell death, respectively. As a whole, these findings show that $\mathrm{SkE}$ also exhibited high activity against the B-Raf V600E mutation.
A

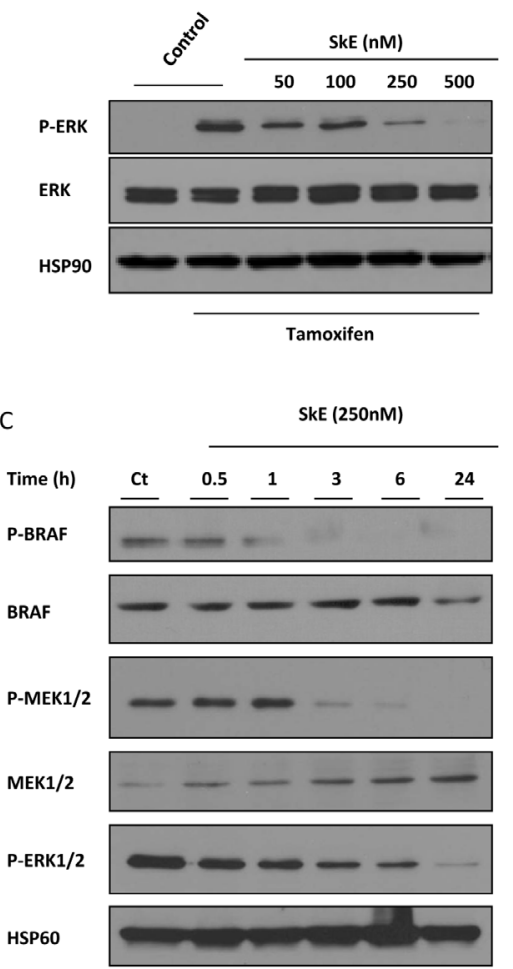

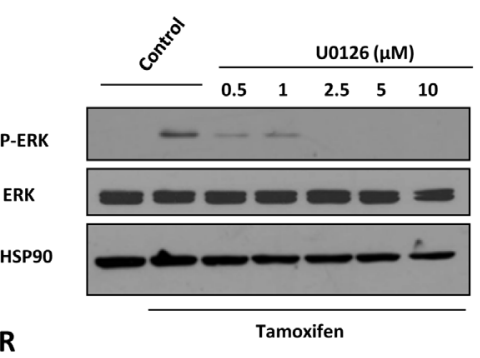

D
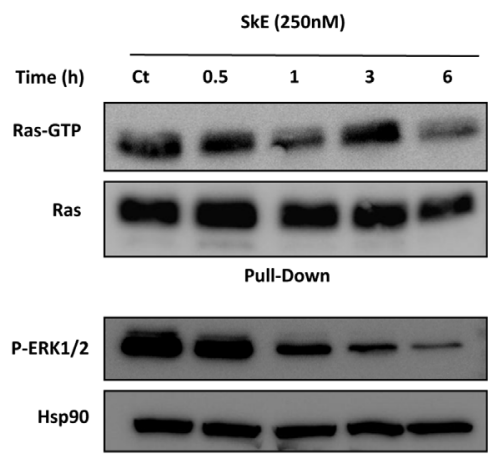

Blot

E

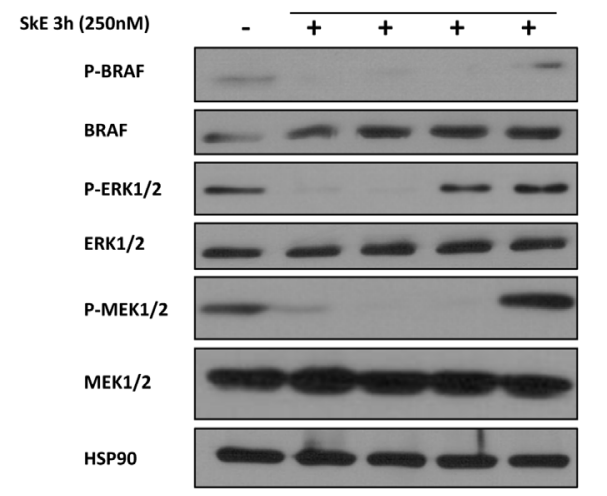

Figure 3: SkE can inhibit the RAF/MEK/ERK signaling pathway. HEK Raf-ER cells were pre-treated with increasing doses of $\mathrm{SkE}$ (A) or U0126 (B) for $1 \mathrm{~h}$. Cells were then treated with tamoxifen $(1 \mu \mathrm{M})$ for one additional hour. Protein samples were separated by electrophoresis, and the expression of Phospho-ERK and ERK was visualized on a Western blot. (C) K562 leukemic cells were treated for different times with $250 \mathrm{nM}$ SkE. The status of phosphorylation of BRAF, MEK and ERK was visualized by Western blot. (D) K562 cells were incubated at $37^{\circ} \mathrm{C}$ with $250 \mathrm{nM} \mathrm{SkE}$ for the indicated times. Ras activity was determined after GST-pull-down. Ras-GTP levels were determined using GST-c-Raf RBD to pull down active GTP-bound Ras from cell extracts by glutathione beads. The beads were washed 4 times and subjected to SDS/PAGE (12\% polyacrylamide). Ras and Phospho-ERK1/2 proteins were detected by Western blot analysis. (E) K562 cells were treated with $250 \mathrm{nM} \mathrm{SkE}$ for $3 \mathrm{~h}$. Cells were then washed and placed in fresh medium for $1 \mathrm{~h}, 3 \mathrm{~h}$ or $24 \mathrm{~h}$. The BRAF, MEK and ERK1/2 protein levels and their phosphorylation status were analyzed by Western blot. HSP60 and HSP90 were used as the loading controls. 


\section{SkE inhibits the growth of CML cells in athymic mice}

To address the efficacy of SkE in vivo, we investigated the ability of the drug to inhibit the growth of the K562 CML cell line implanted in athymic mice. To this end, K562 cells carrying the luciferase gene were injected in the flanks of athymic mice. Mice were randomized and separated into three groups. When tumors reached 100 $\mathrm{mm}^{3}$ in size (after 5 days), each subgroup of mice was treated daily with an intraperitoneal injection of vehicle, $60 \mathrm{mg} / \mathrm{kg}$ imatinib or $1 \mathrm{mg} / \mathrm{kg}$ of SkE. At day 18, imatinib and $\mathrm{SkE}$ had induced tumor regression to a similar extent (Figure 5A). The tumor size was evaluated by photon imaging at days $3,9,14,16$ and 18 following the injection of $30 \mathrm{mg} / \mathrm{kg}$ of luciferin (Figure 5B). The inhibitory effect of SkE on K562 cell growth in vivo was detected as early as 14 days after the onset of injection. By days 16 and 18 , there was almost complete regression of tumors in the imatinib and SkE-treated mice. Finally, histological slides of tumors clearly showed dephosphorylation of ERK in tumors collected from SkE-treated mice at day 18 (Figure 5C). Clearly, there was also a visible decrease in the number of K562 cells present in the tumors of SkEtreated animals. Taken collectively, these data demonstrate that $\operatorname{SkE}(1 \mathrm{mg} / \mathrm{kg})$ is as effective as imatinib $(60 \mathrm{mg} / \mathrm{kg})$, the leading compound for treating CML patients, which is used to inhibit CML cell growth in vivo. Moreover, the effect of SkE in vivo relied on ERK1/2 dephosphorylation.

\section{DISCUSSION}

The Ras/Raf/MEK/ERK cascade is a very attractive target in cancer therapy. Indeed, numerous solid and hematopoietic tumors exhibit activation of this pathway following genetic alterations either in upstream signaling molecules, such as receptor tyrosine kinases and oncogenic fusion proteins, or in overexpression of one of the elements of this pathway $[2,3]$. The upstream regulator of the cascade, Ras, is mutated in 20 to $30 \%$ of human cancers. Of note, the frequency of K-Ras mutations is

A
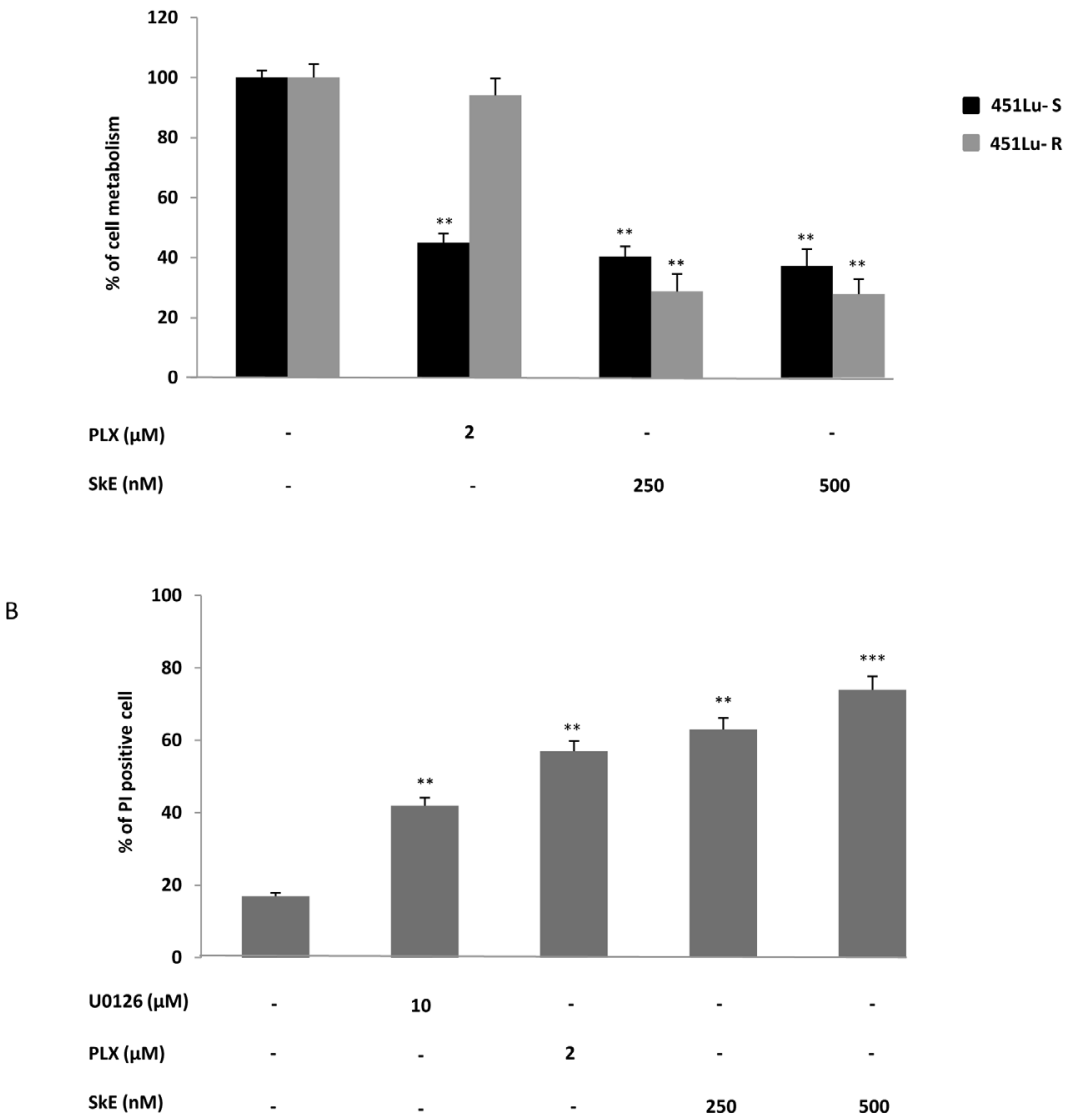

Figure 4: SkE induces cell death of tumors exhibiting the BRAF V600E mutation. (A) PLX-sensitive and PLX-resistant 451Lu melanoma cell lines were treated with either $2 \mu \mathrm{M}$ PLX or increasing doses of SkE. Forty-eight hours later, cell metabolism was measured by the XTT assay as described in the Materials and Methods section. (B) Primary blood cells from patients suffering from hairy cell leukemia were treated with $2 \mu \mathrm{M}$ PLX, $10 \mu \mathrm{M}$ U0126 or increasing doses of SkE. Twenty-four hours later, cells were stained using propidium iodide, and cell death was analyzed with a cytometer. 
very high in advanced pancreatic cancers [23]. Mutations in the downstream kinase B-Raf are also frequently found in cancers. This is well exemplified in melanoma, in which B-Raf mutations are present in $50-70 \%$ of patients, and in $\mathrm{HCL}$, in which the frequency of B-Raf mutations is close to $100 \%$. In both cases, the B-Raf-V600E mutation is consistently detected. The downstream kinase MEK $1 / 2$ is mutated at a low frequency in some cancers, and, to date, there is no evidence of mutation in the downstream kinase ERK1/2. In addition to mutations in various elements of the cascade, the Ras/Raf/MEK/ERK pathway is found to be activated in a very large number of hematopoietic
A

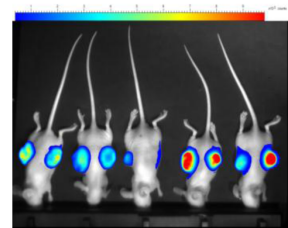

PBS

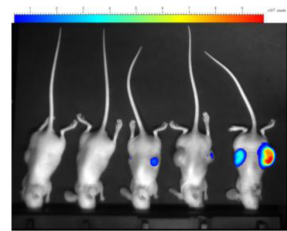

Ima (60mg/kg)

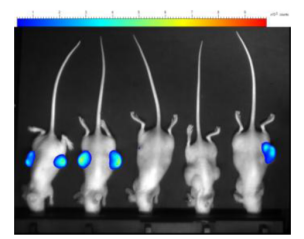

SkE (1mg/kg)

B
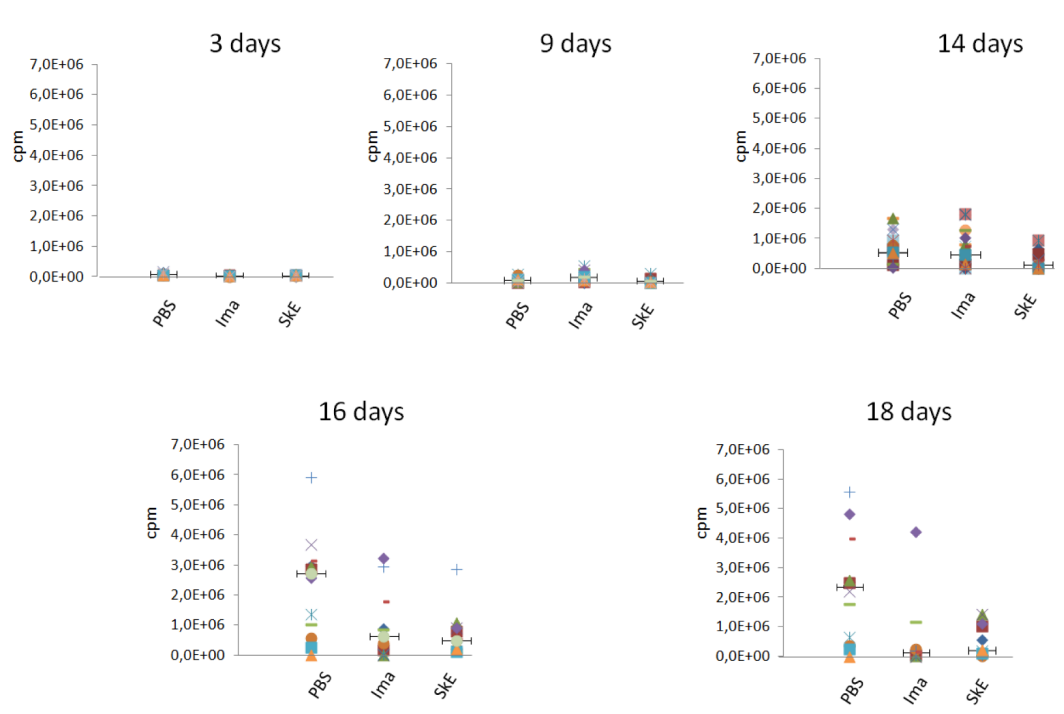

C
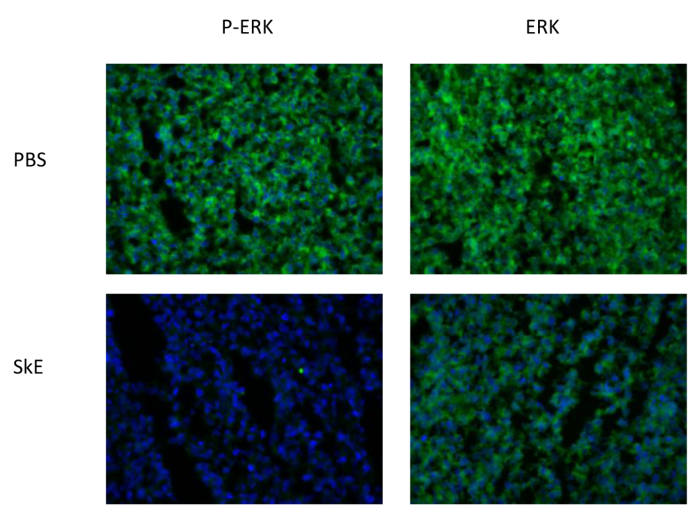

$\mathrm{Ab}$

Figure 5: Chronic treatment with SkE is as effective as imatinib in inhibiting the growth of tumors derived from CML cells in athymic mice. A total of $5 \times 10^{6} \mathrm{~K} 562$ leukemic cells were implanted in both flanks in athymic mice. After tumor establishment, when the tumors reached $100 \mathrm{~mm} 3$, animals received a daily intraperitoneal injection of vehicle, imatinib (60 mg/kg body weight) or $\mathrm{SkE}$ (1 mg/kg body weight). (A) This picture shows the size of tumors at day 16 post injection with vehicle (left panel), Imatinib (middle panel) and SkE (right panel). (B) This panel shows the evolution of tumor growth, expressed in cpm at different times post-injection of the same treatments. (C) Tumors were removed, frozen and cut to perform immune staining. Slides containing a representative section of each tumor were incubated with either an anti-phospho-ERK1/2 or anti-ERK1/2 antibody. Slides were finally mounted and analyzed under a fluorescence microscope. 
and solid tumors [2, 24, 25]. For instance, constitutive activation of the pathway is also observed independent of activating mutations in solid and hematopoietic malignancies, including CML, in which the BCR-ABL fusion oncoprotein (which drives the pathology) is responsible for ERK1/2 activation. Importantly, our group and others have also reported a high constitutive activation of ERK1/2 in several tyrosine kinase inhibitor-resistant CML cell lines [26-28]. Of note, SkE was also found to be highly effective at killing these tyrosine kinase inhibitorresistant CML cell lines (data not shown).

The present study was conducted to determine whether SkE could be used as an antitumoral agent. We show here that very low doses of SkE efficiently inhibited the growth of several solid and hematopoietic cancer cell lines, including melanoma and CML cell lines. Of note, SkE was highly efficient in cells exhibiting constitutive activation of the Ras/Raf/MEK/ERK pathway. For instance, SkE was found to be ten-fold more effective in melanoma cell lines carrying the V600E B-Raf mutation than in melanoma cell lines that did not carry a mutation (Supplementary Figure 1). In addition, SkE was also very potent in CML cell lines exhibiting constitutive activation of the MAP kinase pathway following the expression of the BCR ABL fusion protein.

To decipher the mechanism of action of SkE, we used both melanoma and CML cell lines and primary cells from patients diagnosed with melanoma and HCL and demonstrated that low concentrations of SkE interfere with the activation of ERK, as shown by the clear inhibition of ERK1/2 phosphorylation. We next sought to identify the specific point in the Ras/Raf/MEK/ERK pathway affected by SkE in different cellular models with either constitutive or inducible MAPK kinase activation. Importantly, SkE impaired the activities of both MEK and B-Raf. By contrast, SkE failed to affect Ras activity, strongly suggesting that the drug acts at the level of B-Raf to inhibit the ERK pathway. At present, it is not known whether SkE also affects the activity of other Raf isoforms, including A-Raf and C-Raf.

Importantly, chloroquine, an anti-malarial drug with a chemical structure different from $\mathrm{SkE}$, has previously been reported to inhibit ERK activation [29]. However, the chloroquine dose necessary to achieve complete inhibition of ERK in human peripheral blood monocytes in this study was 1000 times higher than the one used in the present study for SkE in melanoma and CML cell lines. This very high potency of SkE in inhibiting B-Raf prompted us to assess its activity in melanoma cell lines carrying B-RafV600E mutations and in primary cells from HCL patients who consistently carried this mutation. SkE potently inhibited the growth and clonogenic potential of both cell lines, confirming the very potent anti-tumoral effect of this drug, particularly in cells exhibiting constitutive activation of the Ras/Raf/MEK/ERK cascade.

Owing to its ability to inhibit lysosomal protease, chloroquine is often used as an inhibitor of autophagy, a catabolic process that can favor cell survival in adverse conditions, such as cellular stress and nutrient deprivation [30]. In this line, the inhibition of autophagy can sensitize cancer cell lines to chemotherapy, and several clinical trials have been initiated that include chloroquine as a second-line therapeutic agent in different forms of cancers $[31,32]$. However, the findings presented herein clearly establish that an optimal concentration of $\mathrm{SkE}$ failed to affect the lipidation of LC3, arguing against an effect of SkE on autophagy induction when used as a single drug.

In the present study, we also demonstrated that $\mathrm{SkE}$ drastically reduced the growth of CML cells in athymic mice. A dose as low as $1 \mathrm{mg} / \mathrm{kg}$ of SkE was sufficient to inhibit the growth of K562 cells, whereas $60 \mathrm{mg} / \mathrm{kg}$ of imatinib mesylate, the leading treatment for CML, was required to obtain a similar effect. These results clearly show that SkE has an excellent in vivo bioavailability in mice. Moreover, our results strongly suggest that the antiproliferative and proapoptotic effects of $\mathrm{SkE}$ are intimately linked to its ability to interfere with the MAP kinase cascade. This was confirmed by our analysis of tumor histological slides from athymic mice grafted with K562 CML cell lines, which clearly showed a complete inhibition of ERK1/2 phosphorylation in SkE-treated mice.

Finally, we also present evidence that SkE is highly effective at circumventing dabrafenib (GSK2118436) resistance in melanoma cell lines. Dabrafenib is a potent B-Raf inhibitor currently used in phase III studies for metastatic melanoma. It has been reported that dabrafenib initially induced complete remission in patients with metastatic melanoma [14]. However, following this initial beneficial response, all of the patients relapsed. Relapses are likely due to the reactivation of the MAPK pathway and, accordingly, MEK inhibitors such as U0126 can efficiently resensitize dabrafenib-resistant cell lines in vitro. Our group and others have recently reported that the B-Raf inhibitor vemurafenib (PLX-4720) is very effective in HCL patients who carry the B-Raf V600E mutation, inducing complete remission and the restoration of normal blood cell counts and hemoglobin concentration in patients with refractory HCL [11]. Another important finding of the present study is that low concentrations of SkE can inhibit the growth of primary cells from HCL patients more efficiently than vemurafenib (i.e., inhibition occurs in the high nanomolar range versus the micromolar range).

In conclusion, we describe here for the first time the unusual ability of the new compound SkE to inhibit B-Raf activation not only in melanoma and HCL but also in CML cell lines exhibiting constitutive activation of the ERK pathway. In addition, we show that this drug is highly effective at inhibiting HCL-patient-derived primary blood cells carrying this mutation and at inhibiting melanoma cell line with acquired resistance to the B-Raf inhibitors PLX-4720 and GSK2118436. Finally, we also show 
evidence that $\mathrm{SkE}$ at very low doses is highly effective in a preclinical murine model of CML. Collectively, our findings show that $\mathrm{SkE}$ could be a new weapon in the armamentarium of drugs targeting cancers that exhibit constitutive activation of the ERK pathway and that SkE warrants testing in humans.

\section{MATERIALS AND METHODS}

\section{Reagents and antibodies}

RPMI 1640 and DMEM media as well as fetal calf serum (FCS) were purchased from Lonza (Walkersville, MD, USA). Sodium fluoride, orthovanadate, phenylmethylsulfonyl fluoride, aprotinin and leupeptin were purchased from Sigma (Saint-Louis, MO, USA). Imatinib was purchased from Enzo Life Sciences (Villeurbanne, France). U0126 was purchased from Tocris (Bristol, UK). PLX-4720 was purchased from Selleck Chemicals (Houston, TX, USA). Anti-C-Abl, antiMEK1/2, anti-Hsp90 and anti-Hsp60 antibodies were purchased from Santa Cruz Biotechnology (Tebu-Bio, Le Perray en Yvelines, France). Anti-phospho-Abl (Tyr245), anti-phospho-STAT5, anti-phospho-Crkl (Tyr207), antiPARP, anti-phospho-S6 Ribosomal Protein (Ser235/236), anti-S6 Ribosomal Protein, anti-phospho-ERK1/2, anti-ERK1/2, anti-phospho-MEK1/2, anti- phosphoB-Raf, anti-B-Raf and anti-LC3b were purchased from Cell Signaling Technology (Danvers, MA, USA). HRP conjugated anti-mouse, anti-rabbit and anti-goat antibodies were purchased from Dakopatts (Glostrup, Denmark).

\section{Cell lines}

The human CML K562 cell line was provided by ATCC and was grown at $37^{\circ} \mathrm{C}$ under $10 \% \mathrm{CO} 2$ in RPMI 1640 medium supplemented with 5\% FCS (Gibco BRL, Paisley, UK) and 50 units $/ \mathrm{ml}$ of penicillin, $50 \mu \mathrm{g} / \mathrm{ml}$ streptomycin and $1 \mathrm{mM}$ sodium pyruvate. $293 \mathrm{RAF} / \mathrm{ER}$ cells are a derivative of HEK-293 (ATCC: CRL1573) cells that stably express a fusion protein comprising the catalytic domain of Raf-1 and the hormone-binding domain of the estrogen receptor. $293 \mathrm{RAF} / \mathrm{ER}$ cells were cultured in DMEM without phenol red, supplemented with $10 \%$ heat inactivated FCS, as described previously [17]. The 451Lu melanoma cells, which are sensitive or resistant to PLX-4720, were grown in DMEM supplemented with $10 \% \mathrm{FCS}$.

\section{Measurement of cell metabolism (XTT)}

Cells $\left(15 \times 10^{3}\right.$ cells $\left./ 100 \mu \mathrm{l}\right)$ were incubated with the different effectors for the times indicated. A total of 50 $\mu 1$ of XTT reagent (sodium 3'-[1-(phenylaminocarbonyl)3,4-tetrazolium]-bis(4-methoxy-6-nitro)benzene sulfonic acid hydrate) was added to each well. Absorbance of the formazan dye produced by metabolically active cells was measured at $490 \mathrm{~nm}$ as described previously [18]. Each assay was performed in quadruplicate.

\section{Western Blot}

Western blot analyses have been previously described in detail [19].

\section{Phosphoprotein array analysis}

K562 cells were treated (or not treated) with 250 nM SkE for 2 hours. Cells were rinsed with cold PBS and lysed as described for Western blot analysis. Cell lysates were clarified by centrifugation $(10,000 \mathrm{~g}$ for 5 minutes at $4^{\circ} \mathrm{C}$ ), and the protein levels were normalized using the Bradford assay. Then, $150 \mu \mathrm{g}$ of cell extracts was left on the chip as described in the RTK Pathscan array kit from Cell Signaling Technology (Danvers, MA, USA). After incubation and successive steps of washing, the arrays were dried and imaged using a Fujifilm LAS-3000 Imaging System. Duplicate spot intensities were quantified from each array image using the Image $J$ quantification software (U.S. National Institutes of Health, Bethesda, MD, USA).

\section{Primary cell isolation}

Blood samples were collected from patients newly diagnosed with CML or HCL as part of an institutionally approved cellular sample collection protocol. Informed consent was obtained according to institutional guidelines. Mononuclear cells were isolated from blood samples by density centrifugation (Ficoll-Paque ${ }^{\mathrm{TM}}$ Plus), washed with $\mathrm{PBS}, 5 \% \mathrm{SVF}$, and $2 \mathrm{mM}$ EDTA, and then resuspended in cell culture medium (IMDM, 10\% fetal bovine serum) and incubated overnight at $37^{\circ} \mathrm{C}$ in a $5 \% \mathrm{CO} 2$ incubator. CML cells were labeled with CD34 microbeads isolated by magnetic positive selection (StemSep ${ }^{\text {TM }}$ Human CD34 Selection Kit; StemCell, Vancouver, BC, Canada). Purity was estimated to be at least $90 \%$ by FACS analysis. Experiments were performed using an IMDM (10\% fetal bovine serum) for CML and HCL cells.

\section{Colony formation assay}

SkE was added to the K562 CML cell lines $\left(10^{3}\right.$ cells $\left./ \mathrm{ml}\right)$ growing in semi-solid methylcellulose medium. MethoCult H4100 was used for cell lines (StemCell Technologies Inc., Vancouver, Canada). Colonies were detected after 10 days of culture by 
adding $1 \mathrm{mg} / \mathrm{ml}$ of 3-(4,5-dimethylthiazol-2-yl)-2,5diphenyltetrazoliumbromide (MTT) reagent and were scored by Image J quantification software (U.S. National Institutes of Health, Bethesda, MD, USA).

\section{Microscopic analysis of tumors}

Tumors from control or SkE-treated mice were removed, frozen and cut in preparation for immunostaining. Slides containing a representative section of each tumor were fixed, permeabilized and incubated with anti-phospho-ERK $1 / 2$ or anti-ERK antibodies diluted in PBS and 1\% BSA at RT for $1 \mathrm{~h}$. Then, cells were washed and incubated with a secondary anti-Rabbit antibody. Finally, DAPI was used to label the nuclei, and the slides were mounted and then analyzed under a fluorescence microscope (LEICA TCR5500, Leica, Somls, Germany).

\section{Tumor regression experiments in athymic Mice}

Female athymic NMRI Mice (Janvier, Le Genest Saint Ile, France) were randomized into 3 experimental groups, each containing 7 animals. Two sets of animals received a $200 \mu 1$ injection of $5.10^{6} \mathrm{~K} 562$-Luc leukemia cells in both flanks. When tumors reached $100 \mathrm{~mm}^{3}$, the animals were injected intraperitoneally with vehicle (PBS), Imatinib or SkE at dose levels of $60 \mathrm{mg} / \mathrm{kg}$ and $1 \mathrm{mg} / \mathrm{kg}$ body weight, respectively. The growth of the leukemic cells comprising the tumor was visualized in the animal at different days after intraperitoneal injection of $30 \mathrm{mg} / \mathrm{kg}$ luciferin (Caliper Life Sciences) by bioluminescence imaging with a Photon Imager (Biospace $\mathrm{Lab}$ ), as described elsewhere [20]. The in vivo study was conducted according to French legislation on laboratory animal use and care $\left(\mathrm{N}^{\circ} 2001-464\right)$.

\section{Measurement of cell death}

Following U0126, PLX-4720 or SkE treatment, HCL cells were stained with propidium iodide, and the stained cells were analyzed with a cytometer.

\section{Extraction and purification of simalikalactone $\mathbf{E}$}

SkE was extracted and purified from Quassia amara as previously described [1].

\section{Pull-down of the activated form of Rho GTPase}

$\mathrm{K} 562$ cells were incubated at $37^{\circ} \mathrm{C}$ with $250 \mathrm{nM}$ SkE for the indicated times. Ras activity was determined after GST-pull-down. Ras-GTP levels were determined using GST-c-Raf RBD to pull down active GTP-bound Ras from cell extracts by glutathione beads. The beads were washed 4 times and subjected to SDS/PAGE (12\% polyacrylamide). Ras and Phospho-ERK1/2 proteins were detected by Western blot analysis as described previously [21].

\section{Statistical analysis}

All data are presented as the mean $\pm \mathrm{SD}$ of at least three independent determinations. P-values were determined using the Prism V5.0b software (GraphPad, La Jolla, CA, USA). Unless stated otherwise in the figure legend, comparisons of the different groups were made with the one-way ANOVA test with Bonferroni correction. P-values of $0.05(*), 0.01(* *)$ and $0.001(* *)$ were considered statistically significant.

\section{ACKNOWLEDGMENTS}

We are indebted to Drs. M. Herlyn and J. Villanueva (Wistar Institute, Philadelphia, PA, USA) for the kind gift of the $451 \mathrm{Lu}$ melanoma cells that are sensitive and resistant to GSK2118436 and PLX-4720. We also acknowledge Dr. Gilles Pages for the kind gift of the $293 \mathrm{RAF} / \mathrm{ER}$ cells. We acknowledge the C3M Imaging Core Facility (MICA) and genomic facility. We thank the Conseil Regional PACA and the Conseil Général des Alpes-Maritimes for their financial support to C3M. We also acknowledge the Département de la valorisation de I'IRD for financial and legal advice and support.

\section{GRANT SUPPORT}

This work was supported by grant from the Ligue Nationale Contre le Cancer "Equipe labellisée 20112013". GR and AJ are recipients of fellowships from the Fondation de France and the Ligue Nationale Contre le Cancer respectively.

\section{REFERENCE}

1. Cachet N, Hoakwie F, Bertani S, Bourdy G, Deharo E, Stien D, Houel E, Gornitzka H, Fillaux J, Chevalley S, Valentin A, Jullian V. Antimalarial activity of simalikalactone E, a new quassinoid from Quassia amara L. (Simaroubaceae). Antimicrob Agents Chemother. 2009; 53:4393-4398.

2. Steelman LS, Franklin RA, Abrams SL, Chappell W, Kempf CR, Basecke J, Stivala F, Donia M, Fagone P, Nicoletti F, Libra M, Ruvolo P, Ruvolo V, Evangelisti C, Martelli AM, McCubrey JA. Roles of the Ras/Raf/MEK/ERK pathway in leukemia therapy. Leukemia. 2011; 25:1080-1094.

3. Roberts PJ, Der CJ. Targeting the Raf-MEK-ERK mitogenactivated protein kinase cascade for the treatment of cancer. Oncogene. 2007; 26:3291-3310. 
4. Colombino M, Capone M, Lissia A, Cossu A, Rubino C, De Giorgi V, Massi D, Fonsatti E, Staibano S, Nappi O, Pagani E, Casula M, Manca A, Sini M, Franco R, Botti G et al. BRAF/NRAS mutation frequencies among primary tumors and metastases in patients with melanoma. J Clin Oncol. 2012; 30:2522-2529.

5. Sensi M, Nicolini G, Petti C, Bersani I, Lozupone F, Molla A, Vegetti C, Nonaka D, Mortarini R, Parmiani G, Fais S, Anichini A. Mutually exclusive NRASQ61R and BRAFV600E mutations at the single-cell level in the same human melanoma. Oncogene. 2006; 25:3357-3364.

6. Wellbrock C, Hurlstone A. BRAF as therapeutic target in melanoma. Biochem Pharmacol. 2010; 80:561-567.

7. Badalian-Very G, Vergilio JA, Degar BA, MacConaill LE, Brandner B, Calicchio ML, Kuo FC, Ligon AH, Stevenson KE, Kehoe SM, Garraway LA, Hahn WC, Meyerson M, Fleming MD, Rollins BJ. Recurrent BRAF mutations in Langerhans cell histiocytosis. Blood. 2010; 116:1919-1923.

8. Puxeddu E, Moretti S, Elisei R, Romei C, Pascucci R, Martinelli M, Marino C, Avenia N, Rossi ED, Fadda G, Cavaliere A, Ribacchi R, Falorni A, Pontecorvi A, Pacini F, Pinchera A et al. BRAF(V599E) mutation is the leading genetic event in adult sporadic papillary thyroid carcinomas. J Clin Endocrinol Metab. 2004; 89:2414-2420.

9. Tie J, Gibbs P, Lipton L, Christie M, Jorissen RN, Burgess AW, Croxford M, Jones I, Langland R, Kosmider S, McKay D, Bollag G, Nolop K, Sieber OM, Desai J. Optimizing targeted therapeutic development: analysis of a colorectal cancer patient population with the BRAF(V600E) mutation. Int J Cancer. 2011; 128:2075-2084.

10. Tiacci E, Trifonov V, Schiavoni G, Holmes A, Kern W, Martelli MP, Pucciarini A, Bigerna B, Pacini R, Wells VA, Sportoletti P, Pettirossi V, Mannucci R, Elliott O, Liso A, Ambrosetti A et al. BRAF mutations in hairy-cell leukemia. N Engl J Med. 2011; 364:2305-2315.

11. Tiacci E, Schiavoni G, Forconi F, Santi A, Trentin L, Ambrosetti A, Cecchini D, Sozzi E, Francia di Celle P, Di Bello C, Pulsoni A, Foa R, Inghirami G, Falini B. Simple genetic diagnosis of hairy cell leukemia by sensitive detection of the BRAF-V600E mutation. Blood. 2012; 119:192-195.

12. Verma S, Greaves WO, Ravandi F, Reddy N, Bueso-Ramos CE, O'Brien S, Thomas DA, Kantarjian H, Medeiros LJ, Luthra R, Patel KP. Rapid detection and quantitation of BRAF mutations in hairy cell leukemia using a sensitive pyrosequencing assay. Am J Clin Pathol. 2012; 138:153156.

13. Emery CM, Vijayendran KG, Zipser MC, Sawyer AM, Niu L, Kim JJ, Hatton C, Chopra R, Oberholzer PA, Karpova MB, MacConaill LE, Zhang J, Gray NS, Sellers WR, Dummer R, Garraway LA. MEK1 mutations confer resistance to MEK and B-RAF inhibition. Proc Natl Acad Sci U S A. 2009; 106:20411-20416.

14. Sosman JA, Kim KB, Schuchter L, Gonzalez R, Pavlick AC, Weber JS, McArthur GA, Hutson TE, Moschos SJ,
Flaherty KT, Hersey P, Kefford R, Lawrence D, Puzanov I, Lewis KD, Amaravadi RK et al. Survival in BRAF V600mutant advanced melanoma treated with vemurafenib. $\mathrm{N}$ Engl J Med. 2012; 366:707-714.

15. Su F, Bradley WD, Wang Q, Yang H, Xu L, Higgins B, Kolinsky K, Packman K, Kim MJ, Trunzer K, Lee RJ, Schostack K, Carter J, Albert T, Germer S, Rosinski J et al. Resistance to selective BRAF inhibition can be mediated by modest upstream pathway activation. Cancer Res. 2012; 72:969-978.

16. Dietrich S, Glimm H, Andrulis M, von Kalle C, Ho AD, Zenz T. BRAF inhibition in refractory hairy-cell leukemia. N Engl J Med. 2012; 366:2038-2040.

17. Luciano F, Jacquel A, Colosetti P, Herrant M, Cagnol S, Pages G, Auberger P. Phosphorylation of Bim-EL by Erk1/2 on serine 69 promotes its degradation via the proteasome pathway and regulates its proapoptotic function. Oncogene. 2003; 22:6785-6793.

18. Puissant A, Grosso S, Jacquel A, Belhacene N, Colosetti P, Cassuto JP, Auberger P. Imatinib mesylate-resistant human chronic myelogenous leukemia cell lines exhibit high sensitivity to the phytoalexin resveratrol. FASEB J. 2008; 22:1894-1904.

19. Robert G, Puissant A, Dufies M, Marchetti S, Jacquel A, Cluzeau T, Colosetti P, Belhacene N, Kahle P, Da Costa CA, Luciano F, Checler F, Auberger P. The caspase 6 derived N-terminal fragment of DJ-1 promotes apoptosis via increased ROS production. Cell Death Differ. 2012; 19:1769-1778.

20. Puissant A, Dufies M, Fenouille N, Ben Sahra I, Jacquel A, Robert G, Cluzeau T, Deckert M, Tichet M, Cheli Y, Cassuto JP, Raynaud S, Legros L, Pasquet JM, Mahon $\mathrm{FX}$, Luciano $\mathrm{F}$ et al. Imatinib triggers mesenchymallike conversion of CML cells associated with increased aggressiveness. J Mol Cell Biol. 2012; 4:207-220.

21. Munro P, Flatau G, Doye A, Boyer L, Oregioni O, Mege JL, Landraud L, Lemichez E. Activation and proteasomal degradation of rho GTPases by cytotoxic necrotizing factor-1 elicit a controlled inflammatory response. J Biol Chem. 2004; 279:35849-35857.

22. Villanueva J, Vultur A, Lee JT, Somasundaram R, Fukunaga-Kalabis M, Cipolla AK, Wubbenhorst B, Xu X, Gimotty PA, Kee D, Santiago-Walker AE, Letrero R, D'Andrea K, Pushparajan A, Hayden JE, Brown KD et al. Acquired resistance to BRAF inhibitors mediated by a RAF kinase switch in melanoma can be overcome by cotargeting MEK and IGF-1R/PI3K. Cancer Cell. 2010; 18:683-695.

23. McCubrey JA, Steelman LS, Chappell WH, Abrams SL, Montalto G, Cervello M, Nicoletti F, Fagone P, Malaponte G, Mazzarino MC, Candido S, Libra M, Basecke J, Mijatovic S, Maksimovic-Ivanic D, Milella $M$ et al. Mutations and Deregulation of Ras/Raf/MEK/ERK and PI3K/PTEN/Akt/mTOR Cascades. Oncotarget. 2012; 3:954-987.

24. Steelman LS, Chappell WH, Abrams SL, Kempf RC, Long 
J, Laidler P, Mijatovic S, Maksimovic-Ivanic D, Stivala F, Mazzarino MC, Donia M, Fagone P, Malaponte G, Nicoletti F, Libra M, Milella M et al. Roles of the Raf/MEK/ERK and PI3K/PTEN/Akt/mTOR pathways in controlling growth and sensitivity to therapy-implications for cancer and aging. Aging (Albany NY). 2011; 3:192-222.

25. Chappell WH, Steelman LS, Long JM, Kempf RC, Abrams SL, Franklin RA, Basecke J, Stivala F, Donia M, Fagone P, Malaponte G, Mazzarino MC, Nicoletti F, Libra M, Maksimovic-Ivanic D, Mijatovic S et al. Ras/Raf/MEK/ ERK and PI3K/PTEN/Akt/mTOR inhibitors: rationale and importance to inhibiting these pathways in human health. Oncotarget. 2011; 2:135-164.

26. Grosso S, Puissant A, Dufies M, Colosetti P, Jacquel A, Lebrigand K, Barbry P, Deckert M, Cassuto JP, Mari B, Auberger P. Gene expression profiling of imatinib and PD166326-resistant CML cell lines identifies Fyn as a gene associated with resistance to BCR-ABL inhibitors. Mol Cancer Ther. 2009; 8:1924-1933.

27. Fenouille N, Puissant A, Dufies M, Robert G, Jacquel A, Ohanna M, Deckert M, Pasquet JM, Mahon FX, Cassuto JP, Raynaud S, Tartare-Deckert S, Auberger P. Persistent activation of the Fyn/ERK kinase signaling axis mediates imatinib resistance in chronic myelogenous leukemia cells through upregulation of intracellular SPARC. Cancer Res. 2010; 70:9659-9670.

28. Donato NJ, Wu JY, Stapley J, Gallick G, Lin H, Arlinghaus R, Talpaz M. BCR-ABL independence and LYN kinase overexpression in chronic myelogenous leukemia cells selected for resistance to STI571. Blood. 2003; 101:690698.

29. Weber SM, Chen JM, Levitz SM. Inhibition of mitogenactivated protein kinase signaling by chloroquine. J Immunol. 2002; 168:5303-5309.

30. Rubinsztein DC, Gestwicki JE, Murphy LO, Klionsky DJ. Potential therapeutic applications of autophagy. Nat Rev Drug Discov. 2007; 6:304-312.

31. Gupta A, Roy S, Lazar AJ, Wang WL, McAuliffe JC, Reynoso D, McMahon J, Taguchi T, Floris G, DebiecRychter M, Schoffski P, Trent JA, Debnath J, Rubin BP. Autophagy inhibition and antimalarials promote cell death in gastrointestinal stromal tumor (GIST). Proc Natl Acad Sci U S A. 2010; 107:14333-14338.

32. Bellodi C, Lidonnici MR, Hamilton A, Helgason GV, Soliera AR, Ronchetti M, Galavotti S, Young KW, Selmi T, Yacobi R, Van Etten RA, Donato N, Hunter A, Dinsdale $\mathrm{D}$, Tirro E, Vigneri $\mathrm{P}$ et al. Targeting autophagy potentiates tyrosine kinase inhibitor-induced cell death in Philadelphia chromosome-positive cells, including primary CML stem cells. J Clin Invest. 2009; 119:1109-1123. 